\title{
Identification of severe coronary stenosis by two-dimensional strain in acute coronary syndrome without ST segment elevation.
}

\author{
Andrea de Andrade Vilela*, Jorge Eduardo Assef, Elizabete Silva Santos, Fernando Rui Ramos, \\ David Costa de Souza Le Bihan, Rodrigo Bellio de Mattos Barretto, Amanda Guerra de Moraes Rego Sousa \\ Department of Echocardiography, Dante Pazzanese Cardiology Institute, 500 Dr. Dante Pazzanese Avenue, Ibirapuera, \\ São Paulo, Brazil
}

\begin{abstract}
Background: The main objective of our study was to identify, by means of global longitudinal strain (GLS), territorial strain (TS), and postsystolic shortening (PSS) of left ventricle, which patients with acute coronary syndrome without $S T$ elevation (NSTE-ACS) had $\geq 70 \%$ coronary stenosis.

Methods: One hundred patients (PTS) with diagnosis of NSTE-ACS were stratified according to GRACE risk score, and underwent coronary angiography. GLS and TS were calculated. We also evaluated the strain curves in the 18 segments to identify the presence of the PSS and to calculate the post systolic index (PSI).

Results: Mean age was $60 \pm 11.4,62 \%$ was male. The majority were low and moderate cardiovascular risk. They were divided into group A (34 PTS) with coronary stenosis $<70 \%$ and group B (66 PTS) with coronary stenosis $\geq 70 \%$. Clinical score was higher in group $B($ GRACE $=88.7 \pm 24.18, p=0.040)$ and 98. 5\% was in low/moderate risk by GRACE score. GLS allowed the identification of PTS with coronary stenosis $\geq 70 \%$ in this group $(\mathrm{AUC}=\mathbf{0} . \mathbf{7 2}, \mathrm{p}=\mathbf{0} . \mathbf{0 0 1}$, sensitivity $=\mathbf{5 8 \%}$, specificity $=\mathbf{8 8 \%}$, positive predictive value $=75.1 \%$ and negative predictive value $=74.9 \%$ ). Regarding the TS, the accuracy to determine coronary stenosis $\geq 70 \%$ was $0.70(p=0,001)$. The accuracy of the PSS in detecting coronary stenosis $\geq 70 \%$ was $69.3 \%$, with sensitivity estimated at $73.3 \%$ and specificity at 60. $7 \%$.

Conclusion: The GLS, territorial strain, and PSS may improve the detection of severe coronary stenosis in patients with low/moderate risk by GRACE risk score. Thus, it can be an additional tool for a better stratification of such patients in the emergency unit.

Keywords: Acute coronary syndrome, Speckle-tracking, Myocardial strain, Emergency department.
\end{abstract}

Accepted on June 26, 2020

\section{Introduction}

Patients with acute coronary syndrome without ST segment elevation (NSTE-ACS) have a wide spectrum of severity, which varies according to clinical and laboratory characteristics [1]. For this reason, early risk stratification, using clinical scores, is considered Class I recommendation, which enables the probability estimate of adverse cardiovascular events in order to determine the best treatment strategy. In addition, it enables a more cost-effectiveness approach during patients' treatment [2-4]. The Global Registry of Acute Coronary Events (GRACE) risk score is the most usual one, and their prognostic value was established by prospective cohort studies [5].

The GRACE risk score has been shown to have good ability to assess risk for death in patients presenting with acute coronary syndrome, with eight factors based on the characteristic clinical presentation and biomarker results of the patients. It enables the stratification of patients with low, moderate, and high cardiovascular risk. Two-dimensional longitudinal strain enables the quantification of global and territorial myocardial deformity by tracking natural "acoustic tags" in the heart muscle by ultrasound, with lower absolute values in the presence of myocardial ischemia [6,7]. Quantitative assessment of longitudinal deformation measures and myocardial velocity have allowed identification of even subtle changes in the contractile function, such as post systolic shortening [8]. Studies have shown that post systolic shortening has been reported useful for detection of acute ischemia and superior to traditional parameters, such as wall thickening $[9,10]$.

There are few reports in the literature that tested the usefulness of two-dimensional longitudinal strain in patients with NSTEACS as a diagnostic tool. These studies have shown that this method may predict coronary occlusion (100\% stenosis) or identify patients without significant coronary lesions $(<50 \%$ stenosis) [11-13].

The main objective of our study was to identify, by means of global longitudinal strain (GLS), territorial strain (TS), and post systolic shortening (PSS) of left ventricle, which patients with NSTE-ACS had $\geq 70 \%$ coronary stenosis.

\section{Methods}

\section{Population study}

This prospective observational study was approved through the Research Ethics Committee of the Dante Pazzanese Cardiology 
Citation: Vilela ADA, Assef JD, Santos ES, et al. Identification of severe coronary stenosis by two- dimensional strain in acute coronary syndrome without ST segment elevation. Curr Trend Cardiol 2020;4(1):1-9.

Institute under protocol number 3522, and all participants previously agreed with and signed an informed consent form.

One hundred and thirty-three consecutive patients diagnosed with NSTE-ACS and stratified according GRACE risk score were enrolled at the Emergency Department of Dante Pazzanese Cardiology Institute. Inclusion criteria were: 1) Age $>18$ years; 2) A clinical diagnosis of NSTE-ACS; 3) Clinical indication of coronary angiography. Exclusion criteria were: 1) Time between the pain and echocardiographic examination $\geq 48$ hours; 2 ) myocardial thickness $\geq 13 \mathrm{~mm} ; 3$ ) At least one of the following EKG changes-left bundle branch block, atrial fibrillation, and complex ventricular arrhythmias; 4) Prior myocardial infarction; 5) Severe valve dysfunction [14]; 6) Changes in the diagnosis after echocardiographic examination; 7) Inadequate acoustic window. Patients were evaluated and treated according to current guidelines [1-15].

The GRACE risk score was calculated on the basis of age, heart rate, systolic blood pressure, Killip class, cardiac arrest, ST segment deviation, serum creatinine level, and biomarker status from data collected on admission [5]. Patients were divided according to the result of cardiac catheterization: group A-patients with coronary stenosis $<70 \%$; and group B-patients with $\geq 70 \%$ coronary stenosis.

\section{Echocardiographic examination}

All echocardiography examinations were performed within 48 hours upon arrival in the emergency room and before catheterization. All exams were performed with the patient in the left lateral decubitus position using a commercially available system (Vivid 7, General Electric Vingmed Ultrasound Medical System) equipped with a 3. $0-\mathrm{MHz}$ transducer phased-array. Standard two-dimensional images triggered to the QRS complex were saved in digital clips (using an average of three consecutive cycles), and recorded on CDs for further analysis on a workstation (EchoPAC PC® version 11. 0/GE Vingmed Ultrasound).

We evaluated the diastolic function with pulsed Doppler of mitral flow and tissue Doppler. Categorized as normal diastolic function and as altered diastolic function (grade I, II, III and indeterminate) according to the current guidelines [16]. The left atrial volume was calculated according to the biplane arealength measurement and divided by surface body area [17].

The thickness of the septum and posterior wall, relative wall thickness, left ventricular mass index, ejection fraction (EF) by Simpson's biplane method, and wall motion score were calculated. The model of 17 myocardial segments was considered to calculate the wall motion score as follows: normal $=1$; hypokinetic $=2 ;$ akinetic $=3 ;$ dyskinetic $=4$. Wall motion score index (WMSI) was calculated by the sum of scores divided by the number of analyzed segments $[17,18]$.

\section{Two-dimensional longitudinal strain}

Global left ventricular longitudinal strain was quantified using Automatic Function Imaging ${ }^{\circledR}$ (AFI) [19]. For this purpose, one single cardiac cycle is needed from each apical view (apical long-axis, 4-chamber and 2-chamber views).

Peak systolic strain was defined as the maximum value of the peak negative strain during systole. The frame rate of the obtained images was always between 50-90 fps. First, to define the systolic period, the closure of the aortic valve was marked using pulsed Doppler trace of left ventricle outflow tract (LVOT) as a reference. Automatic software measured the time interval between $\mathrm{R}$ wave and aortic valve closure. This interval was used as a reference for the apical long axis, 4-chamber and 2-chamber view loops. After defining the mitral annulus and left ventricular apex with 3 index points at the end-systolic frame in each apical view, the automated algorithm traced 3 concentric lines on the endocardial border, mid-myocardial layer, and epicardial border, including the entire myocardial wall. The tracking algorithm followed the endocardium from this single frame throughout the cardiac cycle allowing a further manual adjustment of the region of interest to ensure that all myocardial regions were included throughout the cardiac cycle. The left ventricle was divided into segments in each apical view, and the tracking quality was validated for each segment (Figure 1).

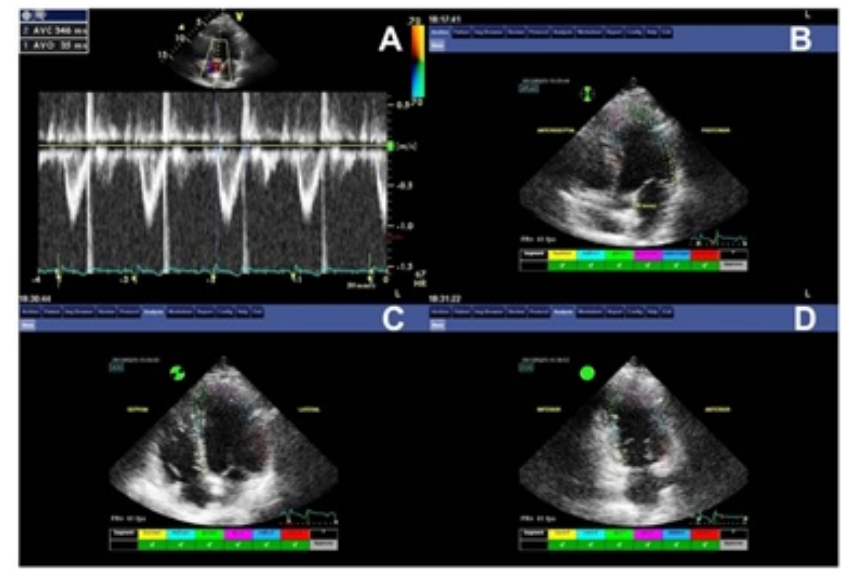

Figure 1. The technique for obtaining longitudinal strain was applied as follows: a-marking the systolic event through pulsed Doppler aortic flow; $b, c$ and d-determining the three points of the endocardial border in three apical planes (long axis, $4 c$ and 2c). The deformation of each of the 17 myocardial segments was calculated by the Automatic Function Imaging ${ }^{\circledR}$ (AFI), subsequently, providing the overall deformation of the left ventricle.

Then, the myocardial motion was analyzed by speckle-tracking within the region of interest. The automated algorithm, using a 17-segment model, provided the peak systolic longitudinal strain for each left ventricular segment in a "bull's eye" plot, with the average value of peak systolic longitudinal strain for each view and the averaged longitudinal peak systolic strain for the complete left ventricle: global longitudinal strain (GLS) (Figure 2). 


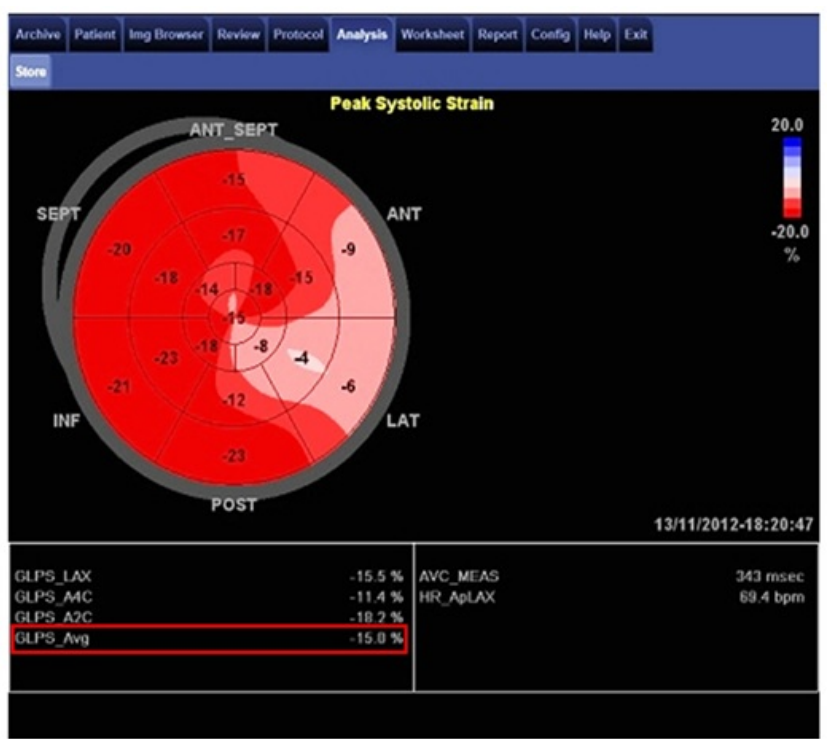

Figure 2. The software provides the peak systolic longitudinal strain for each left ventricular segments in a "bull's eye" plot and the averaged global longitudinal peak systolic strain (GLS) for the complete left ventricle (red rectangle).

For the diagnosis of the probable culprit artery involved in the acute event, we have considered territorial strain (TS) as the average of peak systolic strain values in segments belonging to the theoretical perfusion territory of each major coronary artery on the basis of a 17-segment model, similarly to previous studies [11-13]. The lowest TS absolute value for each patient was assessed as a marker for identification of significant coronary stenosis.

Two-dimensional strain has also been used to investigate postsystolic shortening (PSS), useful for detecting significant coronary artery disease and acute ischemia [9-20, 21]. PSS is defined as the late systolic shortening occurring after aortic valve closure [22]. We evaluated the strain curves in the 18 segments to identify the presence of the PSS and to calculate the postsystolic index (PSI). PSI was calculate as follows: $[$ (maximum strain in cardiac cycle-systolic strain)/(maximum strain in cardiac cycle)] $X 100$. When the PSI value was $\geq 20 \%$ in at least one myocardial wall, we considered the presence of PSS for categorical analysis. This cut-off value was based on previous evidence evaluating PSS [23].

\section{Coronary angiography}

Patients underwent cardiac catheterization between 24 and 72 hours after admission. The exams were archived as digital clips and recorded on CDs for further reassessment. Lesions were classified according to degree of stenosis: 0-no lesion; 1discrete lesion (stenosis $<50 \%$ ); 2-moderate lesion (stenosis between 50 and 70\%); 3-severe injury (stenosis $\geq 70 \%$ ).

\section{Statistical methods}

Continuous variables were expressed as mean $\square$ standard deviation whereas the categorical variables as absolute numbers and percentages. Groups were compared using the student t-test, Mann-Whitney test or Fisher's Exact Test whenever appropriate.

Area under the curve, cut-off point, sensitivity, and specificity for identification coronary stenosis $\geq 70 \%$ were determined by receiver operator characteristic (ROC) analysis.

Logistic regression was used to assess variables independently associated with coronary stenosis $\geq 70 \%$. The kappa index was calculated to verify the concordance between GLS and PSS.

The concordance correlation coefficient (CCC) and the bias correction factor $(\mathrm{BCF})$ were calculated to evaluate the interobserver variability.

For all analysis $\mathrm{p}$ value $<0$. 05 was considered significant.

\section{Results}

\section{Clinical and Echocardiographic Data}

One hundred thirty-three patients were enrolled. However, 25 patients were excluded after undergoing echocardiography due to the fact that results showed they had septal thickness $\geq 13$ $\mathrm{mm}$, severe valve dysfunction, and myocardial segments with fibrotic appearance or inadequate acoustic window. Another four patients did not undergo cardiac catheterization, and another four patients had data loss. In the end, 100 patients remained in the study 60 patients with unstable angina and 40 patients with non-ST-segment elevation myocardial infarction.

The mean age of the study population was $60 \pm 11.4$ years, 99\% patients were low and moderate cardiovascular risk according to the GRACE risk score. The majority were male $(62 \%)$ and there was a high prevalence of hypertension $(81 \%)$ in our population. Regarding the degree of coronary stenosis, $48 \%$ of patients had $\geq 70 \%$ lesion in the left anterior descending coronary artery (LAD), $36 \%$ in the circumflex coronary artery (CCA), and $27 \%$ had $\geq 70 \%$ stenosis in the right coronary artery (RCA). Nineteen patients had coronary occlusion. Among those patients, eleven had collateral circulation and the remainder had an occlusion in the distal bed. Group A (coronary stenosis less than 70\%) comprised 34 patients, and group B (stenosis greater or equal to $70 \%$ ) comprised 66 individuals. The clinical characteristics, values of cardiac troponin, and electrocardiographic findings are shown in Table 1. As shown in Table 1, patients in group B had more prevalence of hypertension, had higher risk score, and higher values of troponin I.

Table 1. Clinical characteristics, values of cardiac troponin and electrocardiographic findings of study population.

\begin{tabular}{|l|l|l|l|}
\hline \multicolumn{1}{|c|}{ Variable } & $\begin{array}{c}\text { Group B } \\
\text { (n=66) } \\
\text { Coronary } \\
\text { Coronary } \\
\text { Con=34) } \\
\text { stenosis<70\% } \\
\text { stenosis } \geq \\
\mathbf{7 0 \%}\end{array}$ & $\mathbf{p}$ \\
\hline Age $(\mathrm{y})$ & $57.5( \pm 10.3$ & $\begin{array}{l}60.8( \pm 11 . \\
3)\end{array}$ & 0.16 \\
\hline Male, $\mathrm{n}(\%)$ & $18(52.9 \%)$ & $44(66.7 \%)$ & 0.19 \\
\hline
\end{tabular}


Citation: Vilela ADA, Assef JE, Santos ES, et al. Identification of severe coronary stenosis by two- dimensional strain in acute coronary syndrome without ST segment elevation. Curr Trend Cardiol 2020;4(1):1-9.

\begin{tabular}{|c|c|c|c|}
\hline Diabetes, n (\%) & $11(32.4 \%)$ & $22(33.3 \%)$ & 1 \\
\hline Hypertension, n (\%) & $23(67.6 \%)$ & $58(87.9 \%)$ & 0.029 \\
\hline Smoking, n (\%) & $9(26.5 \%)$ & 0.37 & 0.37 \\
\hline Hypercholesterolemia, n (\%) & $19(55.9 \%)$ & $44(66.7 \%)$ & 0.38 \\
\hline Family history of CAD & $17(50 \%)$ & $24(36.4 \%)$ & 0.2 \\
\hline GRACE risk score & $77.97( \pm 22.4)$ & $\begin{array}{l}\text { 88. } 7( \pm 24 \text {. } \\
\text { 18) }\end{array}$ & 0.04 \\
\hline Troponin I (ng/ml) & $0.71( \pm 2.18)$ & $\begin{array}{l}\text { 1. } 21( \pm 3 . \\
44)\end{array}$ & 0.03 \\
\hline $\begin{array}{l}\text { Symmetrical T-wave inversion, } \\
\text { admittance }\end{array}$ & $7(21.2 \%)$ & $26(78.8 \%)$ & 0.07 \\
\hline $\begin{array}{l}\mathrm{ST} \text { deviation } \geq 0.5 \mathrm{mV} \text {, } \\
\text { admittance }\end{array}$ & $9(28.1 \%)$ & $9(28.1 \%)$ & 0.49 \\
\hline
\end{tabular}

\begin{tabular}{|l|l|l|l|}
\hline Relative wall thickness $(\mathrm{mm})$ & $0.38 \pm 0.05$ & $0.39 \pm 0.04$ & 0.217 \\
\hline LV mass index $\left(\mathrm{g} / \mathrm{m}^{2}\right)$ & $90.53 \pm 22.4$ & $\begin{array}{l}107.88 \pm 28 . \\
87\end{array}$ & 0.003 \\
\hline LA volume index $\left(\mathrm{ml} / \mathrm{m}^{2}\right)$ & $28 \pm 7.10$ & $33.11 \pm 10.68$ & 0.007 \\
\hline Altered Diastolic Function & $25(73.5 \%)$ & $53(80.3 \%)$ & 0.36 \\
\hline EF Simpson & $0.65 \pm 0.070$ & $0.59 \pm 0.12$ & 0.02 \\
\hline WMSI & $\begin{array}{l}1.07[1.02-1 . \\
12]\end{array}$ & $\begin{array}{l}1.25[1.16-1 . \\
34\end{array}$ & 0.007 \\
\hline GLS (\%) & $-17.84 \pm 2.91$ & $-15.09 \pm 3.85$ & 0.001 \\
\hline
\end{tabular}

LV: left ventricular; LA: left atrium; EF: ejection fraction; WMSI: wall motion score index; GLS: global longitudinal strain; TS: Territorial Strain. Data are expressed as mean \pm SD. $\mathrm{P}$ values are from unpaired $\mathrm{t}$ tests; Mann: Whitney $\mathrm{U}$ tests; or Fisher's exact tests.

CAD: Coronary Artery Disease. Data are expressed as mean \pm $\mathrm{SD}$ or number (percentage). $\mathrm{P}$ values are from unpaired $\mathrm{t}$ tests or Fisher's exact tests.

Table 2 shows the stratification of patients according to risk score and coronary stenosis. In patients with severe coronary stenosis $(\geq 70 \%), 98.5 \%$ were classified as low and moderate cardiovascular risk by GRACE risk score.

Table 2. Classification of cardiovascular risk by GRACE risk score of study population.

\begin{tabular}{|l|l|l|l|}
\hline \multicolumn{1}{|c|}{ GRACE risk score } & \multicolumn{1}{|c|}{$\begin{array}{c}\text { Group B } \\
\text { Group A }(\mathbf{n = 3 4 )} \\
\text { Coronary } \\
\text { stenosis }<70 \%\end{array}$} & $\begin{array}{c}\text { Coronary } \\
\text { stenosis } \geq \\
70 \%\end{array}$ & \multicolumn{1}{|c|}{$\mathbf{p}$} \\
\hline$\leq 108$ (low) & $31(91 \%)$ & $52(78.8 \%)$ & 0.29 \\
\hline $109-139$ (moderate) & $3(8.8 \%)$ & $13(19.7 \%)$ & \\
\hline$\geq 140$ (high) & $0(0 \%)$ & $1(1.5 \%)$ & \\
\hline
\end{tabular}

Echocardiographic characteristics in each group are shown in Table 3. The group with coronary stenosis $\geq 70 \%$ showed higher left ventricular mass index and higher left atrium volume index in relation to those without significant coronary stenosis. Besides, EF by Simpson's method was lower in the group with higher coronary involvement, which also had higher values of WMSI and lower absolute values of both GLS and territorial strain. It was possible to calculate PSI in 88 patients. The presence of PSS occurred in 11 (39. 9\%) patients in group A (28 patients) and in $44(73.3 \%)$ patients in group B (60 patients), with a $\mathrm{p}$ value of 0.004 .

Table 3. Echocardiographic and Two-dimensional global longitudinal strain findings in the study population.

\begin{tabular}{|l|l|c|c|}
\hline \multicolumn{1}{|c|}{ Variable } & $\begin{array}{c}\text { Group A }(\mathbf{n = 3 4 )} \\
\text { Coronary } \\
\text { stenosis<70\% }\end{array}$ & $\begin{array}{c}\text { Group B } \\
\text { (n=66) } \\
\text { Coronary } \\
\text { stenosis } \geq \\
\mathbf{7 0 \%}\end{array}$ & $\mathbf{p}$ \\
\hline Septum thickness $(\mathrm{mm})$ & $9.76 \pm 1.51$ & $10.38 \pm 1.08$ & 0.019 \\
\hline Posterior wall thickness $(\mathrm{mm})$ & $9.21 \pm 1.27$ & $9.92 \pm 1.01$ & 0.005 \\
\hline
\end{tabular}

\section{Accuracy of clinical and echocardiographic data to predict severe coronary stenosis}

A ROC curve was built to compare the accuracy of GRACE score, WMSI, Simpson's EF, GLS, and territorial strain to determine the presence of severe coronary stenosis. The accuracy of GRACE score (area under the curve) to identify patients with coronary stenosis $\geq 70 \%$ was 0 . $63(\mathrm{p}=0$. 040). On the other hand, the accuracy of WMSI and Simpson's EF was $0.67(\mathrm{p}=0.007)$ and $0.64(\mathrm{p}=0.021)$, respectively (Figure $3)$.

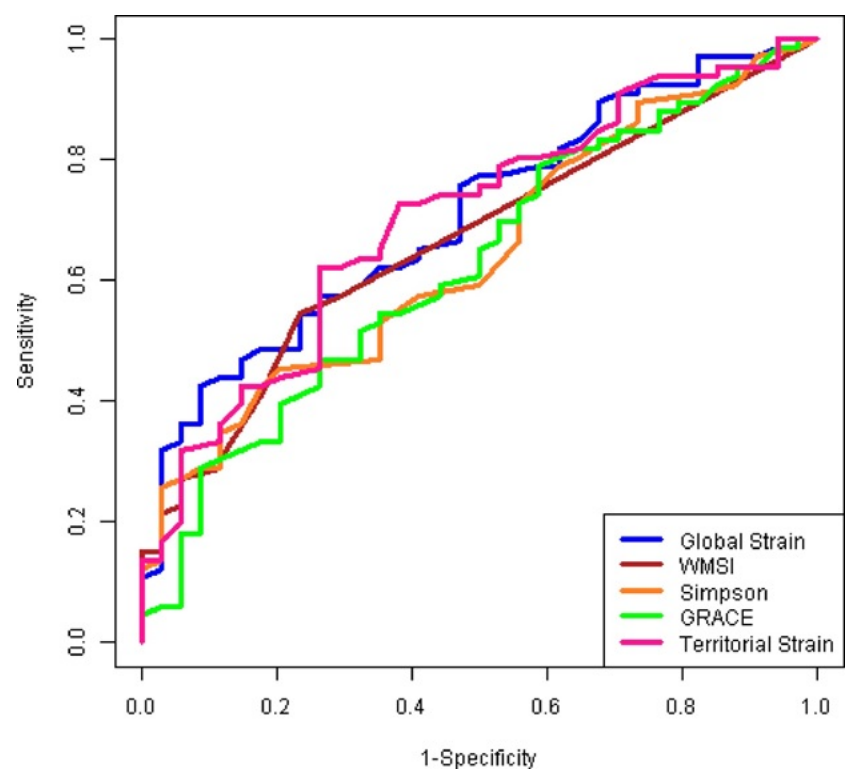

Figure 3. ROC curve analysis for identify coronary stenosis $\geq 70 \%$ in patients with NSTE-ACS. The accuracy of global longitudinal strain was $0.72(p=0.001)$, territorial strain was $0.70(p=0.001), G R A C E$ risk score was $0.63(p=0.040)$ and the accuracy of WMSI and Simpson's EF was 0. $67(p=0.007)$ and $0.64(p=0.021)$, respectively.

Group A had a mean GLS of-17. $84 \pm 2.91$, and group B a mean GLS of-15. $09 \pm 3.85, \mathrm{p}=0.001$. 
The accuracy of GLS was 0.72 and the best cutoff value of- 14 . $9 \%$ was defined, allowing a sensitivity of $58 \%$ and specificity of $88 \%$. According to published data, the prevalence of coronary artery disease in this population was 37. 6\% [24], which allows a positive predictive value of $75.1 \%$ and a negative predictive value of $74.9 \%$.

Regarding the territorial strain, the accuracy to determine coronary stenosis $\geq 70 \%$ was $0.70(p=0,001)$. Group A had a mean TS of $-15.25 \pm 3.59$, and group B a mean TS of- 12. 23 土4. 32 (Table 3 ).

Figure 4 shows two examples of patients with severe coronary lesion on circumflex artery. In case 1, like in other 14 patients of our population, we observed normal values in WMSI and Simpson's EF, with altered GLS. In case 2, like in other 19 patients of our population, we observed only altered TS, with normal values of WMSI, Simpson's EF, and GLS.

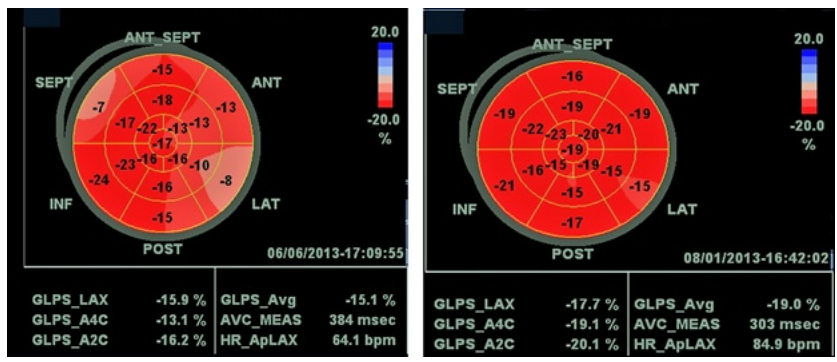

Figure 4. Example of two patients with a severe coronary stenosis: Case 1-50\% in the left anterior descending coronary artery (LAD), $80 \%$ in the circumflex coronary artery (CCA) and $20 \%$ in the right coronary artery (RCA). Simpson's EF was 68\%, WMSI was 1 and GLS was 15. 1\%; Case 2-no lesions in the left anterior descending coronary artery (LAD) and right coronary artery (RCA), $80 \%$ in the circumflex coronary artery (CCA), Simpson's EF was 65\%, WMSI was 1, GLS was 19,9\% and TS was altered in 3 adjacent segments $(-15 \%)$.

The kappa index was 0.58 , which means that there was a moderate agreement between GLS and PSS $(p=0.0001)$. The accuracy of the PSS in detecting coronary stenosis $\geq 70 \%$ was $69.3 \%$, with sensitivity estimated at $73.3 \%$ and specificity at 60. $7 \%$. When we added the PSS to GLS, the discriminative power to detect coronary stenosis $\geq 70 \%$ resulted in an area under the curve of $0.74(\mathrm{p}=0,0004)$, increasing accuracy by $2 \%$ compared to GLS alone (Figure 5 ).

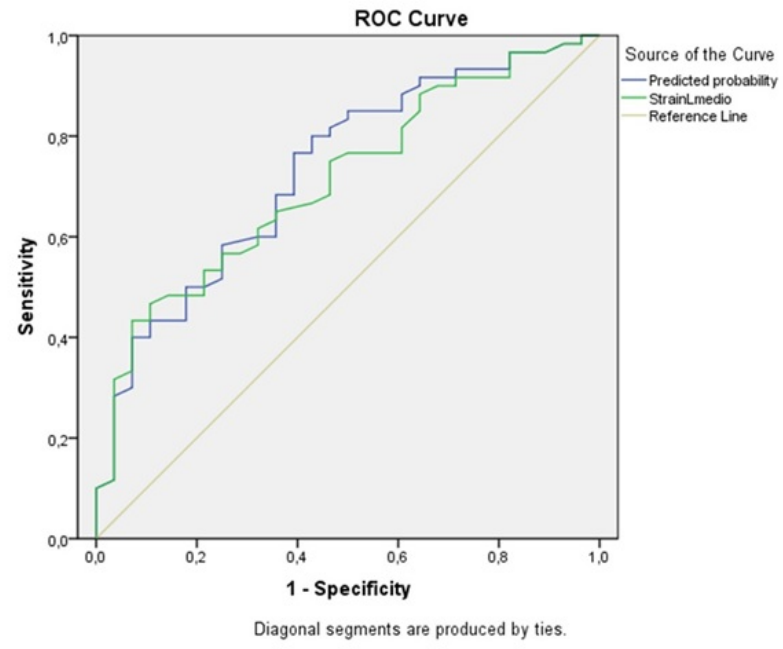

Figure 5. ROC curve analysis for identify coronary stenosis $\geq 70 \%$ in patients with NSTE-ACS. When we added the PSS to GLS, the discriminative power to detect coronary stenosis $\geq 70 \%$ resulted in an area under the curve of 0.74 ( $p=0,0004)$, increasing accuracy by $2 \%$ compared to $G L S$ alone $(A U C=0,72 ; p=0,001)$.

\section{Logistic regression analysis}

To identify variables independently associated with a coronary stenosis $\geq 70 \%$ (group B), three logistic regression models were created. The first model included GLS and possible confounder variables related to hypertensive disease, such as left ventricular mass index, myocardial thickness and previous history of hypertension. In the final model, only GLS emerged as a variable independently associated with coronary lesion $\geq$ $70 \%$ (Table 4).

Table 4. Logistic regression analysis with global longitudinal strain, hypertension, myocardial thickness and left ventricular mass index.

\begin{tabular}{|l|l|l|l|}
\hline \multicolumn{1}{|c|}{ Variable } & \multicolumn{1}{|c|}{ Odss ratio } & \multicolumn{1}{|c|}{$\begin{array}{c}\text { Confidence } \\
\text { interval }\end{array}$} & \multicolumn{1}{|c|}{$\mathbf{p}$} \\
\hline Global longitudinal strain & 1.18 & $1.02-1.39$ & 0.03 \\
\hline Hypertension & 2.45 & $0.74-8.03$ & 0.14 \\
\hline Myocardial thickness & 0.9 & $0.53-1.55$ & 0.72 \\
\hline LV mass index & 1.02 & $0.99-1.05$ & 0.72 \\
\hline
\end{tabular}

\section{LV: Left Ventricular.}

In the other two models created, only variables known to be related to coronary artery disease were included: GRACE risk score, Simpson's EF, WMSI, GLS, territorial strain, and PSS (territorial strain, PSS and GLS were evaluated separately due to collinearity). These analyses demonstrated that both GLS and PSS were independent variables, which allow the identification of coronary stenosis $\geq 70 \%$ (Tables 5-7).

Table 5. Logistic regression analysis with global longitudinal strain, GRACE risk score, EF Simpson and WMSI.

\begin{tabular}{|l|c|c|c|}
\hline \multicolumn{1}{|c|}{ Variables } & Odss ratio & $\begin{array}{c}\text { Confidence } \\
\text { Interval }\end{array}$ & $\mathbf{p}$ \\
\hline Global longitudinal strain & 1.21 & $1.03-1.47$ & 0.04 \\
\hline GRACE risk Score & 1.17 & $0.96-1.42$ & 0.11 \\
\hline EF Simpson & 1 & $0.91-1.09$ & 0.95 \\
\hline WMSI & 1.09 & $0.74-1.61$ & 0.63 \\
\hline
\end{tabular}


Citation: Vilela ADA, Assef JE, Santo ES, et al. Identification of severe coronary stenosis by two-dimensional strain in acute coronary syndrome without ST segment elevation. Curr Trend Cardiol 2020;4(1):1-9.

EF: Ejection Fraction; WMSI: Wall Motion Score Index.

Table 6. Logistic regression analysis with Territorial Strain, GRACE risk score, EF Simpson and WMSI.

\begin{tabular}{|l|l|l|l|}
\hline \multicolumn{1}{|c|}{ Variables } & \multicolumn{1}{|c|}{ Odss ratio } & \multicolumn{1}{c|}{$\begin{array}{c}\text { Confidence } \\
\text { Interval }\end{array}$} & \multicolumn{1}{c|}{$\mathbf{p}$} \\
\hline Territorial Strain & 1.14 & $0.98-1.33$ & 0.086 \\
\hline GRACE risk score & 1.16 & $0.96-1.42$ & 0.12 \\
\hline EF Simpson & 1 & $0.91-1.09$ & 0.98 \\
\hline WMSI & 1.15 & $0.79-1.67$ & 0.46 \\
\hline
\end{tabular}

EF: Ejection Fraction; WMSI: Wall Motion Score Index.

Table 7. Logistic regression analysis with Postsystolic shortening, GRACE risk score, EF Simpson and WMSI.

\begin{tabular}{|l|l|l|l|}
\hline \multicolumn{1}{|c|}{ Variables } & Odss ratio & \multicolumn{1}{|c|}{$\begin{array}{c}\text { Confidence } \\
\text { Interval }\end{array}$} & \multicolumn{1}{|c|}{$\mathbf{p}$} \\
\hline Postsystolic Shortening & 3.55 & $1.24-10.1$ & 0.018 \\
\hline GRACE risk score & 1.02 & $0.99-1.04$ & 0.067 \\
\hline EF Simpson & 0.98 & $0.90-1.08$ & 0.76 \\
\hline WMSI & 2.93 & $0.55-1.56$ & 0.59 \\
\hline
\end{tabular}

EF: Ejection Fraction; WMSI: Wall Motion Score Index.

\section{Analysis of inter observer variability}

Inter observer variability was analyzed by repeating strain and WMSI measurements on 20 randomly selected patients. The concordance correlation coefficient (CCC) was 0.91 and bias correction factor (BCF) 0.97 for the calculation of WMSI and 0.95 (CCC) and 0. 996 (BCF) for global longitudinal strain calculation.

\section{Discussion}

To our knowledge, there are few reports that address the use of two-dimensional longitudinal strain in the emergency department for identification of severe coronary stenosis $(\geq$ $70 \%$ ) in patients categorized as low/moderate cardiovascular risk by GRACE risk score. Published studies have shown that the method was able to predict coronary occlusion $(100 \%$ stenosis) or to identify patients without significant coronary lesion $(\leq 50 \%$ stenosis $)[12,13]$. Guidelines for percutaneous coronary intervention define significant coronary lesion as stenosis $\geq 70 \%$ ( $\geq 50 \%$ in the left main coronary artery), indicating revascularization [25].

Ischemic injury to the myocardium alters regional left ventricular function. However, this modification on myocardial contractility could not be identifiable by standard two- dimensional echocardiography. Speckle tracking echocardiography is a new tool that allows identification of the impaired left ventricular function even when it is not evident by traditional echocardiography [7]. Myocardial deformity (strain) can be evaluated in longitudinal direction, but also in radial and circumferential directions. We have decided to study only longitudinal strain because of two reasons: Firstly, the NSTE-ACS fundamentally affects the subendocardial layer, where longitudinal fibers predominate [26-28]; secondly, the software used for the calculation of two-dimensional strain allows the immediate result only for longitudinal strain; the other deformity directions (circumferential and radial strain) can be calculated only on a workstation through the scanned images. Therefore, longitudinal strain is far more useful in the emergency settings.

The present study showed that GRACE risk score was higher in patients with coronary stenosis $\geq 70 \%$ (Table 1). However, the GRACE risk score failed to differentiate patients according to the level of coronary stenosis when we divided the groups into low, moderate and high risk, as shown in Table 2. In our population, 78 . $8 \%$ of patients with coronary stenosis $\geq 70 \%$ were classified as low risk by GRACE score.

If we consider current guidelines, we can suggest invasive strategy mainly for patients with moderate and high risk [1]. Thus, considering only the GRACE risk score, we would have wrongly discharged $62.6 \%$ of our patients with severe coronary stenosis without treatment (out of 83 patients with low cardiovascular risk, 52 had coronary stenosis $\geq 70 \%$; Table 2). Although there was a correlation between the score value and the severity of coronary stenosis, we can assert that GRACE risk score does not rule out that low-risk patients are free of significant coronary artery disease in our population.

We acknowledge that clinical scores were not designed to differentiate coronary stenosis but to estimate risk. However, it is well known that higher scores are associated with more severe coronary lesions [29,30]. Proving that longitudinal strain is more specific than GRACE to determine a coronary stenosis $\geq 70 \%$ may help to use this tool as a discriminator of patients who would need invasive study earlier.

There are only two studies that evaluated the longitudinal strain in patients with NSTE-ACS, and described the number of risk scores (without stratifying them into low, moderate and high grade) $[12,13]$. Grenne et al. [12] evaluated 111 NSTEACS patients, and divided them into two groups according to the presence (21 patients) or absence of coronary artery occlusion (90 patients). The GRACE risk score was $121 \pm 24$ for the group with coronary occlusion, and $111 \pm 32$ for the group without coronary occlusion. There was no statistical difference between groups [12]. However, Dashlslett et al. [13] studied 64 patients with NSTE-ACS by two-dimensional longitudinal strain to exclude significant coronary lesions (stenosis $>50 \%$ ). They have also found that the GRACE risk score was higher in the group with greater coronary stenosis $(p=0.02)$. They observed a reduction in GLS in patients with NSTE-ACS, similarly to our study. 
The troponin values were higher in group B in our population. However, as the troponin variable is already included in the GRACE risk score, we have not included it in the multivariable analysis, because of collinearity concerns. Furthermore, $40 \%$ of our patients had troponin increase, and therefore, a formal indication of catheterization, according to the current guidelines $[1,15]$. We think that even in such cases, GLS may be useful for demonstrating the "ischemic myocardial extension" of the patient.

Most of our patients were hypertensive (81\%), with different prevalence between groups A and B. National studies confirm the high prevalence of hypertension in our population [24,31]. According to the literature, the longitudinal component of myocardial deformation in hypertensive patients is reduced in direct proportion of the higher left ventricular mass index and greater relative wall thickness [32]. It is also reported that hypertensive patients without pathological increase of left ventricular mass index had similar longitudinal strain compared to patients without hypertension [32]. In our study, hypertensive patients were under treatment and did not have left ventricular hypertrophy. Besides, logistic regression analysis was performed to demonstrate that longitudinal strain correlates only with severe coronary stenosis but not to left ventricular mass index and relative wall thickness. Only GLS remained as an independent variable to identify coronary stenosis $\geq 70 \%$.

Studies that have analyzed the degree of coronary stenosis in patients with NSTE-ACS by GLS also found higher WMSI and lower EF by the Simpson's method in patients with severe coronary artery disease [33]. Those studies along with ours, confirm that such echocardiographic indices could discriminate patients with more severe myocardial damage and, consequently, coronary artery disease. However, according to our findings, we can assert that the GLS is more accurate than $\mathrm{EF}$ and WMSI to discriminate patients with significant coronary stenosis. The logistic regression analysis including those variables could identify only GLS strain as an independent variable. In fact, it is relatively common in clinical practice to find patients with significant coronary disease, normal regional contractility, preserved EF, and normal WMSI, but reduced two-dimensional longitudinal strain.

Our findings are consistent with those described by other authors, who have also observed a reduction in GLS in patients with NSTE-ACS [11-13]. Our cutoff value for GLS (-14. 9\%) was lower than those from other published studies, and as such has allowed a relatively low sensitivity (58\%), but a higher specificity (88\%) for the diagnosis of severe coronary disease in patients with NSTE-ACS, compared with other studies [34]. We think that this difference in our findings, comparing to others, may be explained by the fact that our sample did not exclude patients with previous revascularization, which can result in abnormal myocardial deformation even in absence of significant stenosis. However, this can also improve the external validity of our findings because our population is most likely a population from the "real world".
Grenne et al. reported that PSS was a predictor of coronary occlusion in patients with NSTE-ACS [12]. Recently, Brainin et al. described that PSS allows the diagnosis of coronary stenosis $\geq 70 \%$ in patients with stable angina, in addition to presenting prognostic value, since they correlated with adverse events [20]. Our study, according to previous publications, also showed that PSS predicts significant coronary stenosis in patients with NSTE-ACS. We can say that PSS in association with GLS made the diagnostic power more robust, determined by increasing accuracy by about 2\% (AUC of GLS was 0.72 and AUC of GLS+PSS was 0. 74). In addition, the fact that PSS was more sensitive than GLS (58\% vs. $73.3 \%)$, making this variable useful in the emergency room. In logistic regression analysis PSS was an independent predictor for coronary stenosis $\geq 70 \%$, as was GLS.

Territorial strain was also able to identify patients with coronary stenosis greater than or equal to $70 \%$. Such findings are in accordance with those described in the literature [11-13]. However, the average value we found was lower than reported by others authors. We believe that this is due to the fact that we did not exclude patients with prior revascularization (as described above).

We performed a logistic regression analysis between GRACE, EF, WMSI, and TS to identify coronary stenosis greater than or equal to $70 \%$ and none of the variables was significant. This finding allows us to state that accuracy of GLS and PSS were superior to TS in identifying patients with severe stenosis. That was a rather surprising finding, after all, ischemic heart disease may present with regional LV dysfunction. Besides, intuitively, we would expect TS to be more accurate than GLS, since segments not affected by ischemia were normokinetic or even hyperkinectic. We believe that the possible explanations for such findings are that TS calculation is based on a smaller number of myocardial segments than GLS and there may be individual variation of the territory of anatomical perfusion. These findings are in agreement with those described by Dahlslett et al. [13].

\section{Limitations}

The study of patients referred for cardiac catheterization can be a source of bias, because patients with low and moderate risk who did not undergo catheterization were excluded from our sample. However, this type of bias cannot be avoided in an observational study like ours, since we needed the gold standard in the diagnosis of coronary disease for a proper evaluation of the diagnostic accuracy of the two-dimensional longitudinal strain in patients with NSTE-ACS.

GLS can be abnormal in conditions other than coronary artery disease, which could limit the specificity of this technique in clinical practice. In addition, strain values are still somewhat vendor specific.

The sample is relatively small, despite being in accordance with the calculated minimum number of samples. Larger multicenter studies are required to better evaluate the accuracy of these methods. 
Citation: Vilela ADA, Assef JE, Santos ES, et al. Identification of severe coronary stenosis by two-dimensional strain in acute coronary syndrome without ST segment elevation. Curr Trend Cardiol 2020;4(1):1-9.

\section{Conclusion}

The global longitudinal strain, postsystolic shortening, and territorial strain may improve the detection of severe coronary stenosis in patients with low/moderate risk by GRACE risk score. Thus, it can be an additional tool for a better stratification of such patients in the emergency unit.

\section{Acknowledgements}

We would like to thank the Statistics and Epidemiology Laboratory of Dante Pazzanese Institute of Cardiology, especially JoãoÍtaloFrança MSc, a professional who contributed significantly to the analysis performed in this study.

\section{Funding}

This research did not receive any specific grant from funding agencies in the public, commercial, or not-for-profit sectors.

\section{Informed Consent}

Our Institutional approved this study and the informed consent was obtained from all individual participants included in the study.

\section{References}

1. Anderson JL, Adams CD, Antman EM, et al. 2012 ACCF/AHA 2007 guidelines for the management of patients with unstable angina/non-ST-elevation myocardial infarction: a report of the American College of Cardiology Foundation/American Heart Association Task Force on Practice Guidelines. J Am Coll Cardiol. 2013;61(23):e179-347.

2. Zaacks SM, Liebson PR, Calvin JE, et al. Unstable angina and non-Q wave myocardial infarction: does the clinical diagnosis have therapeutic implications? J Am Coll Cardiol. 1999. 33(1):107-18.

3. Calvin JE, Klein LW, VandenBerg BJ, et al. Clinical predictors easily obtained at presentation predict resource utilization in unstable angina. Am Heart J. 1998;136(3): 373-81.

4. Kong DF, Blazing MA, O'Connor CM. The health care burden of unstable angina. Cardiol Clin. 1999;17(2): 247-61.

5. Granger CB, Goldberg RJ, Dabbous O, et al. Global Registry of Acute Coronary Events Investigators. Predictors of hospital mortality in the global registry of acute coronary events. Arch Interm Med. 2003; 163(19): 2345-53.

6. Amundsen BH, Helle-Valle T, Edvardsen T, et al. Noninvasive myocardial strain measurement by speckle tracking echocardiography; validation against sonomicrometry and tagged magnetic resonance imaging. J Am Coll Cardiol. 2006;47(4):789-93.

7. Choi JO, Cho SW, Song YB, et al. Longitudinal 2D strain at rest predicts the presence of left main and three vessel coronary artery disease in patients without regional wall motion abnormality. Eur J Echocardiogr. 2009;10(5): 695-701.

8. Brainin P, Biering-Soresen SR, Mogechvang R, et al. Postsystolic shortening by speckle tracking echocardiography is an independent predictor of cardiovascular events and mortality in the general population. J Am Heart Assoc. 2018; 7(6):e008367.

9. Kukulki T, Jamal F, Herbots L, et al. Identification of acutely ischemic myocardium using ultrasonic strain measurements: a clinical study in patients undergoing coronary angioplasty. J Am Coll Cardiol. 2003; 41(5): 810-19.

10. Asanuma T, Uranishi A, Masuda K, et al. Assessment of myocardial ischemic memory using persistence of postsystolic thickening after recovering from ischemia. JACC Cardiovasc Imaging. 2009; 2(11): 1253-61.

11. Eek C, Grenne B, Brunvand H, et al. () Strain echocardiography predicts acute coronary occlusion in patients with non-ST-segment elevation acute coronary syndrome. Eur J Echocardiogr. 2010; 11(6):501-08.

12. Grenne B, Eek C, Sjøli B, et al. Acute coronary occlusion in non-ST-elevation acute coronary syndrome: outcome and early identification by strain echocardiography. Heart. 2010; 96(19):1550-56.

13. Dahlslett T, Karlsen S, Grenne S, et al. Early assessment of strain echocardiography can accurately exclude significant coronary artery stenosis in suspect non-ST-segment elevation acute coronary syndrome. J Am Soc Echocardiogr. 2014; 27(5):512-19.

14. Nishimura RA, Otto CM, Bonow RO, et al. AHA/ACC Guideline for the Management of Patients With Valvular Heart Disease: A Report of the American College of Cardiology/American Heart Association Task Force on Practice Guidelines. Circulation. 2014;129(23):2440-92

15. Feitosa-Filho GS, Baracioli LM, et al. SBC Guideline on unstable angina and non ST elevation myocardial infarction: Executive summary. Arq Bras Cardiol. 2015; 105(3): 214-27.

16. Nagueh SF, Smiscth OA, Appleton CP, et al. Recommendation for the evaluation of left ventricular diastolic function by echocardiography: An update from the American Society of Echocardiography and the European Association of Cardiovascular Imaging. J Am Soc Echocardiogr. 2016; 29(4): 277-314.

17. Lang RM, Bierig M, Devereux RB, et al. () Chamber Quantification Writing Group; American Society of Echocardiography's Guidelines and Standards Committee; European Association of Echocardiography. Recommendations for chamber quantification: A report from the American Society of Echocardiography's Guidelines and Standards Committee and the Chamber Quantification Writing Group, developed in conjunction with the European Association of Echocardiography, a branch of the European Society of Cardiology. J Am Soc Echocardiogr. 2005; 18(12):1440-63. 
18. Cerqueira MD, Weissman NJ, Dilsizian V, et al. Standardized myocardial segmentation and nomenclature for tomographic imaging of the heart: A statement for healthcare profissionals from the Cardiac Imaging Committee of the Council on Clinical Cardiology of the American Heart Association. Circulation. 2002;105(4): 539-42.

19. Leitman M, Lysyansky P, Sidenko S, et al. Bidimensional strain: A novel software for real time quantitative echocardiographic assessment of myocardial function. J Am Soc Echocardiogr. 2004; 17(10): 1021-29.

20. Brainin P, Hoffmann S, Fritz-Hansen T, et al. Usefulness of postsystolic shortening to diagnose coronary artery disease and predicts future cardiovascular events in Stable Angina Pectoris. J Am Soc Echocardiogr. 2018; 31(8): 870-79.

21. Okuda K, Asanuma T, Hirano T, et al. Impact of the coronary flow reduction at resto $\mathrm{n}$ myocardial perfusion and functional indices derived from myocardial contrast and strain echocardiography. J Am Soc Echocardiogr. 2006; 19(6): 781-87.

22. Asanuma T, Nakatani S. Myocardial ischaemia and postsystolic shortening. Heart. 2015; 101(7): 509-16.

23. Voight JU, Lindenmeir G, Exner B, et al. Incidence and characteristics of segmental postsystolic longitudinal shortening in normal, acutely ischemic, and scarred myocardium. J Am Soc Echocardiogr. 2003; 16(5): 415-23.

24. Santos ESD, Minuzzo L, Pereira MP, et al. Acute coronary syndrome registry at a cardiology emergency center. Arq Bras Cardiol. 2006; 87(5):597-602.

25. Levine GN, Bates ER, Blankenship JC, et al. American College of Cardiology Foundation; American Heart Association Task Force on Practice Guidelines; Society for Cardiovascular Angiography and Interventions 2011 ACCF/AHA/SCAI Guideline for Percutaneous Coronary Intervention. A report of the American College of Cardiology Foundation/American Heart Association Task Force on Practice Guidelines and the Society for Cardiovascular Angiography and Interventions. J Am Coll Cardiol. 2011;58(24):e44-122

26. Sarafoff N, Schuster T, Vochem R, et al. Association of the ST elevation and non ST elevation presentation on ECG with transmurality and size of myocardial infarction as assessed by contrast-enhanced magnetic resonance imaging. J Electrocardiol. 2013; 46(2):100-06.

27. Geyer H, Caracciolo G, Abe H, et al. (2010) assessment of myocardial mechanics using speckle tracking echocardiography: fundamentals and clinical applications. J Am Soc Echocardiogr. 2010; 23(4):351-69.

28. Collier P, Phelan D, Klein A. A test in context: Myocardial strain measured by Speckle-Tracking Echocardiography. J Am Coll Cardiol. 2017; 69(8):1043-56.

29. Khandelwal G, Jain A, Rathore M. Prediction of angiographic extent of coronary artery disease on the basis of clinical risk scores in patients of unstable angina. J Clin Diagn Res. 2015; 9(11): 13-16.

30. Barbosa CE, Viana M, Brito M, et al. Accuracy of the GRACE and TIMI scores in predicting the angiographic severity of acute coronary syndrome. Arq Bras Cardiol. 2012;99(3): 818-24.

31. Piegas LS, Avezum A, Guimarães $\mathrm{HP}$, et al. Comportamento da síndromecoronarianaaguda. resultados de um registrobrasileiro. Arq Bras Cardiol. 2013; 100:502-10.

32. Narayanan A, Aurigemma GP, Chinali M, et al. Cardiac mechanics in mild hypertensive heart disease: A specklestrain imaging study. Circ Cardiovasc Imaging. 2009;2:382-90.

33. Stein JH, Neumann A, Preston LM, et al. Improved risk stratification in unstable angina: Identification of patients at low risk for in-hospital cardiac events by admission echocardiography. Clin Cardiol. 1998;21:725-30.

34. Norum IB, Ruddox V, Edvardsen T, et al. Diagnostic accuracy of left ventricular longitudinal function by speckle tracking echocardiography to predict significant coronary artery stenosis. A systematic review. BMC Medical Imaging. 2015;15:25.

\section{*Correspondence to}

Andrea de Andrade Vilela

Department of Echocardiography

Dante Pazzanese Cardiology Institute

Dr. Dante Pazzanese avenue

São Paulo

Brazil

E-mail:andrea.vilela@grupofleury.com.br 